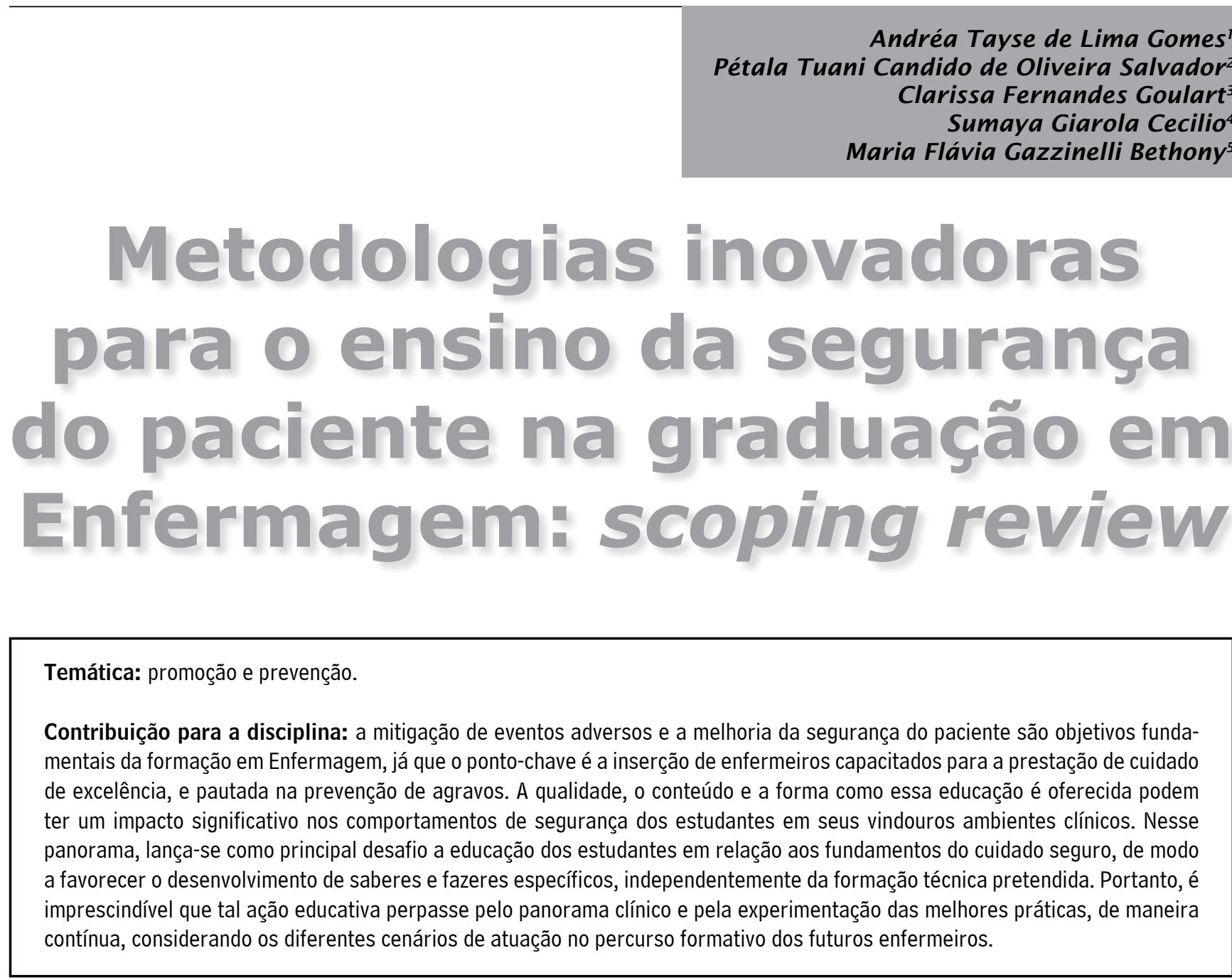

\title{
RESUMO
}

Objetivo: identificar as metodologias inovadoras utilizadas para o ensino da segurança do paciente na graduação em Enfermagem. Materiais e método: scoping review conduzida conforme as recomendações do Joanna Briggs Institute Reviewers' Manual, por meio das bases de dados: Medline/PubMed, Cumulative Index of Nursing and Allied Health, Scopus, Web of Science, Education Resources Information Center, Literatura Latino-Americana e do Caribe em Ciências da Saúde, Catálogo de Teses e Dissertações da Coordenação de Aperfeiçoamento de Pessoal de Nível Superior, The National Library of Australia's, Academic Archive Online, Digital Access to Research Theses Europe E-Theses Portal, Electronic Theses Online Service, Repositório Científico de Acesso Aberto de Portugal, National ETD Portal e Theses Canada. Resultados: foram incluídos 19 estudos; a maioria do tipo descritivo ( $n=8 ; 42,1 \%$ e quase experimental

\section{DOI: 10.5294/aqui.2020.20.1.8}

Para citar este artigo / Para citar este artículo / To reference this article Gomes ATL, Salvador PTCO, Goulart CF, Cecilio SG, Bethony MFG. Innovative Methodologies to Teach Patient Safety in Undergraduate Nursing: Scoping Review. Aquichan 2020;20(1):e2018. DOI: https://doi.org/10.5294/aqui.2020.20.1.8

$1 \bowtie$ https://orcid.org/0000-0003-0505-5783. Universidade Federal de Minas Gerais, Brasil. andreatayse@ufmg.br

2 https://orcid.org/0000-0002-3208-6270. Escola de Enfermagem de Natal, Universidade Federal do Rio Grande do Norte, Brasil.

3 https://orcid.org/0000-0002-9744-8658. Universidade Federal de Minas Gerais, Brasil.

4 https://orcid.org/0000-0002-4571-8038. Universidade Federal de Minas Gerais, Brasil.

5 https://orcid.org/0000-0003-0523-3973. Universidade Federal de Minas Gerais, Brasil.

Recebido: 18/07/2019 Submetido: $15 / 09 / 2019$ Aceito por pares: 29/11/2019 Aceito: 06/12/2019 
( $n=7 ; 36,8 \%)$; com abordagem quantitativa $(n=11 ; 57,9 \%$ ); realizados nos Estados Unidos ( $n=4 ; 21,1 \%)$, em $2013(n=4 ; 21,1 \%)$ e em $2017(n=4 ; 21,1 \%)$. As metodologias inovadoras utilizadas para 0 ensino da segurança do paciente na graduação em Enfermagem que se destacaram foram o cenário de prática assistencial simulada em laboratório $(n=14 ; 73,7 \%)$ e os vídeos educacionais $(n=7$; 36,8 \%). Conclusões: identificou-se que as metodologias inovadoras utilizadas para 0 ensino da segurança do paciente na graduação em Enfermagem foram simulação, vídeos, encenação/dramatização e filmes, todas aplicadas na modalidade de ensino presencial.

PALAVRAS-CHAVE (FonTE: DeCS)

Ensino; segurança do paciente; metodologia; tecnologia educacional; educação em Enfermagem. 


\section{Metodologías innovadoras para la enseñanza de la seguridad del paciente en el pregrado de Enfermería: scoping review}

\section{RESUMEN}

Objetivo: identificar las metodologías innovadoras utilizadas para la enseñanza de la seguridad del paciente en el pregrado de Enfermería. Materiales y método: scoping review realizada de acuerdo con las recomendaciones del Joanna Briggs Institute Reviewers' Manual, por medio de las bases de datos: Medline/PubMed, Cumulative Index of Nursing and Allied Health, Scopus, Web of Science, Education Resources Information Center, Literatura Latinoamericana y del Caribe en Ciencias de la Salud, Catálogo de Tesis de la Coordenação de Aperfeiçoamento de Pessoal de Nivel Superior, The National Library of Australia's, Academic Archive Online, Digital Access to Research Theses Europe E-Theses Portal, Electronic Theses Online Service, Repositório Científico de Acesso Aberto de Portugal, National ETD Portal y Theses Canada. Resultados: se incluyeron 19 estudios; la mayoría de tipo descriptivo $(n=8 ; 42,1 \%$ ) y casi experimental $(n=7 ; 36,8 \%)$; con enfoque cuantitativo $(n=11 ; 57,9 \%)$; realizados en los Estados Unidos $(n=4 ; 21,1 \%)$, en el $2013(n=4 ; 21,1$ $\%$ y el $2017(n=4 ; 21,1 \%)$. Las metodologías innovadoras utilizadas para la enseñanza de la seguridad del paciente en el pregrado de Enfermería que se destacaron fueron: el escenario de práctica asistencial simulada en laboratorio $(n=14 ; 73,7 \%)$ y los videos educacionales $(n=7 ; 36,8 \%$ ). Conclusiones: se identificó que las metodologías innovadoras utilizadas para la enseñanza de la seguridad del paciente en el pregrado de Enfermería fueron simulacro, videos, escenificación/dramatización y películas, todas aplicadas en la modalidad de enseñanza presencial.

PALABRAS CLAVE (Fuente: DeCS)

Enseñanza; seguridade del paciente; metodología; tecnología educacional; educación en Enfermería. 


\section{Innovative Methodologies to Teach Patient Safety in Undergraduate Nursing: Scoping Review}

\section{ABSTRACT}

Objective: This work sought to identify the innovative methodologies used to teach patient safety in undergraduate Nursing. Materials and method: this is a scoping review conducted according to the recommendations by the Joanna Briggs Institute Reviewers' Manual, through the databases: Medline/PubMed, Cumulative Index of Nursing and Allied Health, Scopus, Web of Science, Education Resources Information Center, Latin American and Caribbean Literature on Health Sciences, Catálogo de Tesis de la Coordenação de Aperfeiçoamento de Pessoal de Nivel Superior, The National Library of Australia's Academic Archive Online, Digital Access to Research Theses Europe E-Theses Portal, Electronic Theses Online Service, Repositório Científico de Acesso Aberto de Portugal, National ETD Portal, and Theses Canada. Results: the study included 19 studies, most of descriptive type $(n=8 ; 42.1 \%)$ and quasi-experimental $(n=7$; $36.8 \%)$ with quantative approach $(n=11 ; 57.9 \%)$, conducted in the United States $(n=4 ; 21.1 \%)$, in $2013(n=4 ; 21.1 \%)$ and $2017(n=4$; $21.1 \%$ ). The innovative methodologies used to teach patient safety in undergraduate Nursing that were highlighted included the scenario of the care practice simulated in the laboratory $(n=14 ; 73.7 \%)$ and educational videos $(n=7 ; 36.8 \%)$. Conclusions: it was identified that innovative methodologies used to teach patient safety in undergraduate Nursing were simulation, videos, staging/role playing, and films, all applied in the classroom teaching modality.

\section{KEYWORDS (SOURCE: DeCS)}

Teaching; patient safety; methodology; educational technology; nursing education. 


\section{Introdução}

Desde a publicação do relatório To Err is Human, pelo Institute of Medicine (IOM) dos Estados Unidos da América (EUA), em 1999, a questão da segurança do paciente tem adquirido relevância mundial. 0 documento aponta que, aproximadamente, 100.000 pessoas morrem a cada ano em hospitais norte-americanos, decorrentes de eventos adversos preveníveis (1).

As práticas dos profissionais de saúde têm sido marcadas por elevadas taxas de eventos adversos relacionados a procedimentos cirúrgicos, administração de medicamentos, infecções adquiridas em hospitais, lesões provocadas em pacientes, falhas nos sistemas de atendimento hospitalar, danos permanentes e mortes. Práticas inadequadas, inseguras e negligentes afetam entre 3 e $16 \%$ dos pacientes hospitalizados em países desenvolvidos (2-5).

0 reconhecimento dessa problemática e dos danos não intencionais causados pelos cuidados de saúde tem desencadeado múltiplas reflexões, principalmente, acerca da formação dos profissionais de saúde, que, em alguns momentos, se apresenta fragilizada por não acompanhar o ritmo acelerado de inovações na prática, as mudanças nas condições, nas diversidades e na força de trabalho impostas nas últimas décadas (6).

Nesse contexto, assente-se que a prevenção de eventos adversos e a melhoria da segurança do paciente são importantes aspectos a serem tratados na formação em Enfermagem. A qualidade, o conteúdo e a forma como essa educação é oferecida podem ter um impacto significativo nos comportamentos de segurança dos estudantes em seus futuros ambientes clínicos $(7,8)$.

A segurança do paciente deve ser discutida e, sobretudo, compreendida desde a graduação, lançando mão de metodologias inovadoras capazes de suscitar o pensamento crítico e reflexivo dos estudantes, uma vez que essa perspectiva é abordada pelo Ministério da Saúde brasileiro desde o lançamento da Portaria 529, de 2013, ao relatar, em um dos seus objetivos específicos, a relevância da fomentação da inclusão do tema "segurança do paciente" no ensino técnico, no de graduação e no de pós-graduação na área da saúde (9).

Diante dessa realidade, em 2011, a Organização Mundial de Saúde (OMS) definiu, dentre as suas atividades, aquelas destinadas a promover o ensino relacionado aos princípios do cuidado seguro na formação dos estudantes das áreas da saúde, com 0 objetivo geral de realizar um atendimento clínico centrado no paciente. Como objetivos específicos, foram estabelecidos: 1) preparar os estudantes da área de saúde para uma prática segura; 2) informar as instituições de ensino sobre as principais questões de segurança; 3) aprimorar os aspectos basilares atrelados à segurança do paciente como tema de todos os cursos da área de saúde; 4) fornecer um programa abrangente para auxiliar 0 ensino e a integração da aprendizagem da segurança do paciente; 5) incentivar o desenvolvimento de treinamento na segurança do paciente (10).

Para atingir esses objetivos, é imprescindível que se façam revisões críticas dos currículos para incluir, nos procedimentos aplicados ao ensino-aprendizagem, atividades voltadas à segurança do paciente, a fim de gerar mudanças disciplinares, com ênfase na integração de estratégias que colaborem para 0 aprimoramento de habilidades de raciocínio e para o julgamento clínico dos estudantes $(10,11)$.

Discute-se, dessa forma, a necessidade de alterações nas Diretrizes Curriculares Nacionais (DCN) dos cursos de Enfermagem do Brasil que orientem a formação superior de forma abrangente às evidências contemporâneas sobre a segurança do paciente. Em paralelo à relevância de atualização dos conteúdos curriculares, compreende-se a importância de as Instituições de Ensino Superior acompanharem, também, as inovações pedagógicas que ultrapassam as formas tradicionais de ensino (12-14).

Essas inovações representam não somente a inserção de novidades e tecnologias no ensino, mas também incluem uma mudança na forma de entender o conhecimento. Dentre as características de inovações pedagógicas, destacam-se: a ruptura com a forma tradicional de ensinar e aprender; a participação dos estudantes na definição de percursos e critérios no processo de ensino e aprendizagem; a reconfiguração de saberes, competências e vivências pessoais; a reorganização da relação entre a teoria e a prática; a transição da percepção da concepção, do desenvolvimento e da avaliação da experiência no ensino-aprendizagem $(15,16)$.

As metodologias inovadoras envolvem 0 ato de oferecer, ao fazer pedagógico, novas possibilidades, atitudes e tomadas de decisão em sala de aula. Ao se optar por esse tipo de recurso pedagógico, o educador poderá romper com modelos que simplesmente depositam informações e conhecimentos em seus 
estudantes. Desse modo, faz-se relevante discutir sobre as metodologias de ensino inovadoras que sejam capazes de facilitar a compreensão sobre os pilares da segurança do paciente na formação em Enfermagem $(17,18)$.

Diante disso, esta pesquisa teve como objetivo identificar as metodologias inovadoras utilizadas para o ensino da segurança do paciente na graduação em Enfermagem.

\section{Materiais e método}

A scoping review conduzida conforme as diretrizes do Joanna Briggs Institute Reviewers' Manual, segundo o cenário teórico levantado por autores da área $(19,20)$. As etapas seguidas foram: 1) elaboração da questão norteadora da scoping review; 2) identificação dos estudos pertinentes; 3) triagem dos estudos; 4) análise dos dados coletados e 5) tratamento, síntese e apresentação dos resultados.

Para a estruturação e execução da pesquisa, preparou-se um protocolo que continha informações concernentes ao objetivo do estudo, à população envolvida na pesquisa, à formulação da questão de pesquisa, aos critérios de elegibilidade, à estratégia e bases utilizadas para a coleta de dados, à definição de variáveis para a extração dos dados e à forma como os dados seriam sintetizados.

0 delineamento do objetivo e da questão de pesquisa, bem como a seleção dos descritores, das sinonímias e das palavras-chave utilizadas na coleta de dados seguiu os parâmetros da estratégia P (População), C (Conceito) e C (Contexto). Neste estudo, a população diz respeito às metodologias inovadoras; o conceito está atrelado ao ensino da segurança do paciente, e o contexto é a graduação em Enfermagem. Nesse sentido, a pesquisa foi orientada pelo questionamento: quais metodologias inovadoras têm sido utilizadas para o ensino da segurança do paciente na graduação em Enfermagem?

Inicialmente, foi realizada uma busca preliminar de possíveis estudos semelhantes nas bases de dados JBI COnNECT+, The Cochrane Library e Prospero, em janeiro de 2019, nas quais não foram detectados protocolos e/ou revisões publicados enquadrados em temática análoga.

Em seguida, buscou-se identificar as palavras-chave e as sinonímias disponíveis na literatura por meio da combinação dos
Descritores em Ciências da Saúde (DeCS) — palavras em português - e em Medical Subject Headings (MESH) — termos em inglês - componentes do mnemônico PCC desta pesquisa, em que: (P) Metodologia/Methodology AND Tecnologia educacional/ Educational technology AND (C) Ensino/Teaching AND Segurança do paciente/Patient safety AND (C) Educação em Enfermagem/ Education, Nursing. Esse momento envolveu três bases de dados, a saber: Literatura Latino-Americana e do Caribe em Ciências da Saúde (Lilacs), Medline/PubMed e Cumulative Index of Nursing and Allied Health (Cinahl).

A segunda e a terceira etapas desta scoping review (identificação e seleção dos estudos relevantes) foram executadas de janeiro a fevereiro de 2019, por intermédio das bases de dados Medline/PubMed, Cinahl, Scopus, Web of Science, Education Resources Information Center (Eric), Lilacs, Catálogo de Teses e Dissertações da Coordenação de Aperfeiçoamento de Pessoal de Nível Superior (Capes), The National Library of Australia's (Trove), Academic Archive Online (Diva), Digital Access to Research Theses (Dart), Europe E-Theses Portal, Electronic Theses Online Service (EThOS), Repositório Científico de Acesso Aberto de Portugal (RCAAP), National ETD Portal e Theses Canada, a fim de pesquisar artigos, dissertações e teses que retratassem a utilização de metodologias inovadoras voltadas ao ensino do cuidado seguro na graduação em Enfermagem.

As estratégias de busca utilizadas nessa investigação foram: 1) para as bases de dados em língua portuguesa - (Ensino $O R$ Educação) AND (Segurança do paciente $O R$ Melhoria da qualidade) $A N D$ (Metodologia OR Métodos de ensino) AND (Tecnologia educacional $O R$ Simulação por computador $O R$ Aprendizagem baseada em simulação $O R$ Jogos de vídeo $O R$ Tecnologia de aprendizagem $O R$ Vídeo $O R$ Recurso educacional aberto $O R$ Filmes instrutivos e Vídeo $O R$ Inovações $O R$ Simulação $O R$ Encenação $O R$ Paciente virtual $O R$ Ambiente baseado em simulação) AND (Educação em Enfermagem $O R$ Estudantes de Enfermagem) e 2) para as bases de dados internacionais - (Teaching OR Education) AND (Patient safety OR Quality improvement) AND (Methodology OR Teaching methods) AND (Educational technology OR Computer simulation OR Simulation-based learning OR Video game $O R$ Learning technology $O R$ Video OR Open education resource $O R$ Instructional films and video OR Innovations $O R$ Simulation $O R$ Role-play $O R$ Virtual patient simulation-based environment) AND (Education, Nursing OR Nursing students). 
Os estudos foram selecionados com suporte nos respectivos critérios de elegibilidade: incluíram-se artigos científicos, dissertações e teses; publicados na íntegra em meio on-line; que discorressem sobre a utilização de metodologias inovadoras voltadas ao ensino da segurança do paciente na graduação em Enfermagem. Por sua vez, foram excluídos editoriais, relatos de experiência, ensaios teóricos, reflexões e revisões. Não foi traçado limite temporal na busca realizada.

Preliminarmente, avaliaram-se 0 título e o resumo dos estudos identificados, com fundamentação nos critérios de elegibilidade preestabelecidos; em seguida, as publicações selecionadas foram recuperadas para a leitura completa, conforme o esquema exposto na Figura 1.

Figura 1. Esquema do processo de seleção dos estudos. Belo Horizonte-MG, Brasil, 2019

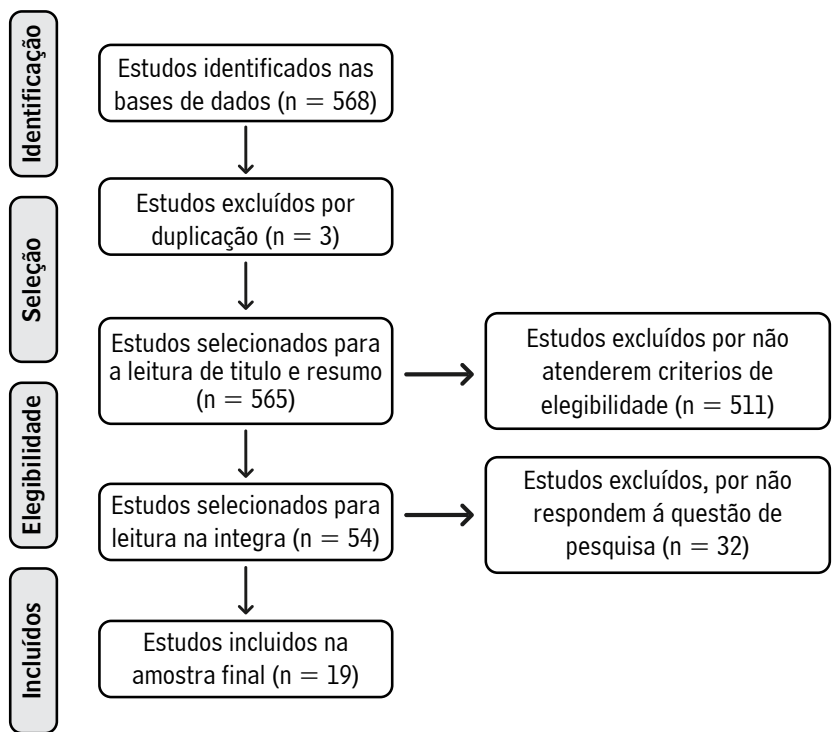

Fonte: Prisma — Fluxograma para scoping review (adaptado) (19).

No que concerne à quarta etapa desta investigação (análise dos dados), procedeu-se à extração dos dados dos estudos incluídos na amostra final em uma planilha construída no Microsoft Excel 2016, de acordo com as variáveis: ano em que o estudo foi publicado, país onde a pesquisa foi realizada, objetivo da investigação, tipo de estudo, abordagem metodológica, nível de evidência (21), metodologia inovadora abordada, modalidade de ensino em que a metodologia foi utilizada, fragilidades e potencialidades inerentes à metodologia inovadora aplicada.
Por fim, os dados foram sintetizados de forma descritiva (n e \%), com a utilização de tabelas e quadros. Igualmente, os estudos foram codificados, a fim de facilitar a compreensão da disposição final dos dados, da seguinte maneira: "E" (Estudo), seguido pelos algarismos arábicos $1,2,3, \ldots 19$, de modo a assumir a representação El, E2, E3, ... E19.

\section{Resultados}

Foram incluídos 19 estudos; a maioria do tipo descritivo $(n=8 ; 42,1 \%)$ e quase experimental $(n=7 ; 36,8 \%)$; com abordagem metodológica quantitativa $(\mathrm{n}=11 ; 57,9 \%)$; realizados nos Estados Unidos $(n=4 ; 21,1 \%)$, na Austrália $(n=3 ; 15,8 \%)$ e na Turquia $(n=3 ; 15,8 \%)$; em $2013(n=4 ; 21,1 \%)$ e em 2017 $(n=4 ; 21,1 \%)$.

0 Tabela 1 descreve a caracterização dos estudos que compuseram a amostra desta scoping review conforme os respectivos códigos identificadores (E1, E2, E3, ... E19), o objetivo do estudo, 0 país e 0 ano em que a pesquisa foi realizada, o tipo de pesquisa, 0 Nível de Evidência (NE) e a abordagem metodológica.

Todos os estudos envolveram a aplicação de metodologias inovadoras para 0 ensino do cuidado seguro aos estudantes da graduação em Enfermagem em sua modalidade presencial, e a inovação usada com maior frequência foi a simulação $(n=14$; 73,7\%), conforme apresentado na Tabela 1.

A Tabela 2 expõe, quantitativamente, as metodologias inovadoras utilizadas, associando-as aos respectivos estudos que fizeram parte do escopo da pesquisa.

Tabela 1. Metodologias inovadoras aplicadas ao ensino da segurança do paciente $(n=19)$. Belo Horizonte-MG, Brasil, 2019

\begin{tabular}{|c|c|c|}
\hline Metodologias utilizadas (códigos dos estudos) & $\mathrm{n}^{6}$ & $\%$ \\
\hline $\begin{array}{c}\text { Cenário de prática assistencial simulada em laboratório } \\
\text { (El, E6, E8-E9, El0-E19) }\end{array}$ & 14 & 73,7 \\
\hline Vídeos educacionais (E2-E4, E7, Ell, El6-El7) & 7 & 36,8 \\
\hline Encenação/Dramatização (E3, E5, El9) & 3 & 15,8 \\
\hline Filmes (E8) & 1 & 5,3 \\
\hline
\end{tabular}

Fonte: elaboração própria.

\footnotetext{
6 Alguns estudos abordaram o uso de mais de uma metodologia.
} 
Tabela 2. Caracterização dos estudos incluídos. Belo Horizonte-MG, Brasil, 2019

\begin{tabular}{|c|c|c|c|}
\hline $\begin{array}{l}\text { Código do estudo } \\
\text { (Referência) }\end{array}$ & Objetivo do estudo & País (ano) & Tipo/NE/abordagem \\
\hline $\mathrm{El}(22)$ & $\begin{array}{l}\text { Avaliar a eficácia do treinamento teórico relacionado a erros médicos e à segurança do paciente e } \\
\text { do treinamento de simulação prática sobre os conhecimentos e níveis de habilidades dos estudantes. }\end{array}$ & Turquia (2018) & $\begin{array}{l}\text { Experimental/1/ } \\
\text { quantitativa }\end{array}$ \\
\hline E2 (23) & $\begin{array}{l}\text { Determinar se o uso de um vídeo simulado por especialistas durante a pré-aprendizagem melhorou o } \\
\text { desempenho de habilidades na simulação. }\end{array}$ & $\begin{array}{l}\text { Estados Unidos } \\
\quad(2018)\end{array}$ & $\begin{array}{l}\text { Quase-experimental } \\
\text { /2/quantitativa }\end{array}$ \\
\hline E3 (24) & $\begin{array}{l}\text { Explorar a avaliação dos estudantes de bacharelado em Enfermagem sobre os métodos de aprendi- } \\
\text { zagem combinados para melhorar as habilidades de comunicação em Enfermagem em saúde mental. }\end{array}$ & Noruega (2018) & $\begin{array}{l}\text { Exploratório/2/ } \\
\text { quantitativa e } \\
\text { qualitativa }\end{array}$ \\
\hline E4 (25) & $\begin{array}{l}\text { Examinar os efeitos do uso de vídeos educativos sobre as habilidades do estudante de Enfermagem } \\
\text { para administrar medicação parenteral. }\end{array}$ & Turquia (2017) & $\begin{array}{l}\text { Quase-experimental } \\
\text { /2/quantitativa }\end{array}$ \\
\hline E5 (26) & $\begin{array}{l}\text { Descrever as respostas de estudantes de graduação de Enfermagem sobre uma experiência de encena- } \\
\text { ção que simulou o gerenciamento de interrupções durante a administração de medicação. }\end{array}$ & Austrália (2017) & $\begin{array}{c}\text { Descritivo/4/ } \\
\text { qualitativa }\end{array}$ \\
\hline E6 (27) & $\begin{array}{l}\text { Descrever como os acadêmicos de Enfermagem apreendem o cuidado voltado a pacientes em situações } \\
\text { agudas por meio de exercícios de simulação, observação e debriefing. }\end{array}$ & Suécia (2017) & $\begin{array}{l}\text { Descritivo/4/ } \\
\text { qualitativa }\end{array}$ \\
\hline E7 (28) & $\begin{array}{l}\text { Avaliar a eficácia de um programa de intervenção educacional no conhecimento de questões básicas } \\
\text { de segurança do paciente em estudantes de graduação de uma escola de Enfermagem privada que } \\
\text { frequentam sua primeira prática clínica. }\end{array}$ & $\begin{array}{l}\text { Chile } \\
(2017)\end{array}$ & $\begin{array}{l}\text { Quase-experimental } \\
\text { /2/quantitativa }\end{array}$ \\
\hline E8 (29) & $\begin{array}{l}\text { Determinar se a exposição simulada a situações de erros muda as atitudes de uma forma que pode ter } \\
\text { um impacto positivo sobre os comportamentos de prevenção de erros. }\end{array}$ & $\begin{array}{l}\text { Estados Unidos } \\
\quad(2016)\end{array}$ & $\begin{array}{l}\text { Quase-experimental } \\
\text { /2/quantitativa }\end{array}$ \\
\hline E9 (30) & $\begin{array}{l}\text { Investigar a viabilidade de gravações audiovisuais para aumentar o feedback após as simulações agu- } \\
\text { das de deterioração do paciente. }\end{array}$ & Austrália (2016) & $\begin{array}{l}\text { Descritivo /4/ } \\
\text { quantitativa }\end{array}$ \\
\hline $\mathrm{El0}(31)$ & $\begin{array}{l}\text { Comparar a eficácia do debriefing oral assistido por vídeo e do debriefing oral sozinho sobre comporta- } \\
\text { mentos por estudantes de Enfermagem de graduação durante a simulação de alta fidelidade. }\end{array}$ & $\begin{array}{l}\text { Estados Unidos } \\
\text { (2014) }\end{array}$ & $\begin{array}{l}\text { Quase-experimental } \\
\text { /2/quantitativa }\end{array}$ \\
\hline Ell (32) & $\begin{array}{l}\text { Avaliar o uso de um modelo de melhores práticas on-line como um complemento ao ensino de habilida- } \\
\text { des clínicas de administração de medicação oral a estudantes de graduação em Enfermagem. }\end{array}$ & Escócia (2013) & Coorte/3/mista \\
\hline El2 (33) & $\begin{array}{l}\text { Explorar os resultados de desenvolvimento e avaliação de um pacote de habilidades simuladas que usam } \\
\text { uma abordagem de aprendizagem baseada em problemas com estudantes de Enfermagem em geral. }\end{array}$ & Irlanda (2013) & $\begin{array}{l}\text { Descritivo/4/quan- } \\
\text { titativa }\end{array}$ \\
\hline El3 (34) & $\begin{array}{l}\text { Avaliar a eficácia de um workshop de habilidades clínicas sobre a administração de medicamentos para } \\
\text { melhorar as habilidades de cálculo de dose-droga de estudantes de Enfermagem do segundo ano, com } \\
\text { o objetivo de promover a segurança na administração de medicamentos. }\end{array}$ & Itália (2013) & $\begin{array}{l}\text { Descritivo/2/quan- } \\
\text { titativa }\end{array}$ \\
\hline El4 (35) & $\begin{array}{l}\text { Determinar se } 0 \text { treinamento em simulação é um método de ensino apropriado e afetivo a ser usado } \\
\text { para o ensino do cuidado seguro em Enfermagem perinatal. }\end{array}$ & Turquia (2013) & $\begin{array}{l}\text { Quase-experimen- } \\
\text { tal/2/quantitativa }\end{array}$ \\
\hline El5 (36) & $\begin{array}{l}\text { Fornecer aos estudantes do terceiro ano do curso de graduação em Enfermagem uma exposição cui- } \\
\text { dadosamente planejada para um cenário que simula uma mudança típica em um ambiente de prática. }\end{array}$ & $\begin{array}{l}\text { País de Gales } \\
\text { (2012) }\end{array}$ & Coorte/3/mista \\
\hline El6 (37) & $\begin{array}{l}\text { Explorar e descrever os modos comunicativos que os estudantes empregam para coordenar a equipe } \\
\text { em um ambiente de simulação projetado para o treinamento da equipe de ressuscitação. }\end{array}$ & Noruega (2011) & $\begin{array}{l}\text { Descritivo/4/qua- } \\
\quad \text { litativa }\end{array}$ \\
\hline El7 (38) & $\begin{array}{l}\text { Investigar os processos utilizados por estudantes de Enfermagem do final do curso para reconhecer e } \\
\text { agir sobre pistas clínicas de deterioração em um ambiente simulado. }\end{array}$ & Austrália (2010) & $\begin{array}{l}\text { Descritivo/4/qua- } \\
\quad \text { litativa }\end{array}$ \\
\hline El8 (39) & $\begin{array}{l}\text { Investigar os recursos de aprendizagem de um programa de simulação de computador CathSim usado } \\
\text { para a ministração de treinamento de habilidades de cateterização intravenosa. }\end{array}$ & Suécia (2010) & $\begin{array}{l}\text { Quase-experimen- } \\
\text { tal/2/quantitativa }\end{array}$ \\
\hline E19 (40) & $\begin{array}{l}\text { Descrever como uma faculdade de Enfermagem começou a integrar o ensino de segurança do paciente } \\
\text { em experiências de simulação para estudantes de graduação em Enfermagem. }\end{array}$ & $\begin{array}{l}\text { Estados Unidos } \\
\qquad(2009)\end{array}$ & $\begin{array}{l}\text { Descritivo/4/qua- } \\
\quad \text { litativa }\end{array}$ \\
\hline
\end{tabular}

Fonte: elaboração própria. 
A Tabela 3 detalha as potencialidades e as fragilidades relacionadas às metodologias inovadoras, abordadas pelos estudos analisados, usadas para o ensino do cuidado seguro aos estudantes em Enfermagem.

Tabela 3. Potencialidades e fragilidades inerentes às metodologias inovadoras utilizadas para 0 ensino da segurança do paciente. Belo Horizonte-MG, Brasil, 2019

\begin{tabular}{|c|c|}
\hline Metodologias inovadoras & $\begin{array}{l}\text { Potencialidades e fragilidades } \\
\text { (códigos dos estudos) }\end{array}$ \\
\hline \multirow{2}{*}{$\begin{array}{l}\text { Cenário de prática assistencial } \\
\text { simulada em laboratório }\end{array}$} & $\begin{array}{l}\text { Potencialidades } \\
\text { - Os estudantes podem praticar as habilidades repetidamente, até que se sintam seguros (E15, El8-E19). } \\
\text { - Permite que os estudantes desenvolvam as habilidades, com integração da teoria e da prática, e refletindo criticamente sobre } \\
\text { seu desempenho, individual e coletivo, em um ambiente seguro (El, El2-E13, Ell, El5). } \\
\text { - Os estudantes tornam-se conscientes quanto às lacunas em seu conhecimento, o que é reconhecido durante as observações e } \\
\text { em grupos de debriefing (E6). } \\
\text { - Permite o treinamento prático dos estudantes por meio de uma aprendizagem ativa e independente, com multimídia interativa } \\
\text { e ferramenta de simulação de fácil manuseio. Os estudantes podem ter a confiança aumentada em relação a uma habilidade } \\
\text { específica (E18). } \\
\text { - Melhora as habilidades de comunicação entre os estudantes (E14). }\end{array}$ \\
\hline & $\begin{array}{l}\text { Fragilidades } \\
\text { - Diferentes e complexos ambientes clínicos, redução do controle sobre as condições ambientais, necessidade de garantir a } \\
\text { segurança do paciente versus necessidades de melhoria das habilidades e estresse podem afetar negativamente o processo } \\
\text { formativo (E1). } \\
\text { - A utilização dessa metodologia no currículo de graduação em Enfermagem é demorada e requer um investimento significativo } \\
\text { em pessoal e recursos (E8, El2). } \\
\text { - Requer uma abordagem cautelosa e cuidadosamente planejada, com tempo suficiente para que cada estudante seja observado e } \\
\text { receba feedback (E3). } \\
\text { - Não é uma abordagem de ensino adequada a todos os estudantes. Esse método não é capaz de trazer benefícios aos participan- } \\
\text { tes nem aumento da confiança para a atuação em ambiente prático real (E15). }\end{array}$ \\
\hline \multirow[t]{2}{*}{ Vídeos educacionais } & $\begin{array}{l}\text { Potencialidades } \\
\text { - Contribuições positivas para o treinamento de habilidades com grandes grupos de estudantes (E2, E4). } \\
\text { - } 0 \text { acesso ilimitado a vídeos on-line como complemento ao ensino da prática clínica está associado a uma maior aquisição de } \\
\text { habilidades e à satisfação do estudante (Ell). }\end{array}$ \\
\hline & $\begin{array}{l}\text { Fragilidades } \\
\text { - } 0 \text { uso pode ser associado aos métodos de ensino tradicionais, sem substituí-los no treinamento prático de habilidades clínicas. } \\
\text { Embora a eficácia do modelo de treinamento apoiado em multimídia esteja bem-estabelecida, seu uso permanece limitado (E4). }\end{array}$ \\
\hline \multirow[t]{2}{*}{ Encenação/Dramatização } & $\begin{array}{l}\text { Potencialidades } \\
\text { - É uma forma válida de ensino e de fácil acesso, que não é apenas agradável para a maioria dos estudantes, mas pode fornecer- } \\
\text { - Ihes as conexões necessárias para usar o pensamento crítico e os julgamentos clínicos sólidos (E5). } \\
\text { - É um método relevante, especialmente no que diz respeito à aprendizagem ativa. A filmagem da dramatização oferece uma } \\
\text { oportunidade única para refletir sobre o próprio desempenho (E3). }\end{array}$ \\
\hline & $\begin{array}{l}\text { Fragilidades } \\
\text { - Não foram apontadas fragilidades quanto ao recurso de encenação/dramatização para o ensino do cuidado seguro aos estudan- } \\
\text { tes de graduação em Enfermagem entre os estudos incluídos na amostra final. }\end{array}$ \\
\hline \multirow[t]{2}{*}{ Filmes } & $\begin{array}{l}\text { Potencialidades } \\
\text { - A educação cinematográfica tornou os participantes mais conscientes quanto às consequências devastadoras dos erros durante } \\
\text { a assistência à saúde. A exposição a erros por meio de filmes pode sensibilizar quanto ao risco daqueles e as possibilidades de } \\
\text { evitá-los (E8-E9). }\end{array}$ \\
\hline & $\begin{array}{l}\text { Fragilidades } \\
\text { - Não foram apontadas fragilidades quanto ao uso de filmes para o ensino de segurança do paciente aos estudantes de graduação } \\
\text { em Enfermagem entre os estudos incluídos na amostra final. }\end{array}$ \\
\hline
\end{tabular}

Fonte: elaboração própria. 
As metodologias inovadoras utilizadas para o ensino da segurança do paciente aos estudantes do ensino superior em Enfermagem favorecem significativamente ao desenvolvimento de habilidades em um ambiente seguro e de forma crítica e reflexiva, desde que a execução da estratégia de ensino seja cuidadosamente planejada, principalmente em termos de tempo, pessoal e recursos materiais.

\section{Discussão}

Destaca-se que a predominância de estudos do tipo descritivo entre as pesquisas analisadas na amostra final dessa scoping review realça a recorrente necessidade de descrever a realidade de determinados fenômenos no meio científico. Entretanto, isso revela a importância da produção investigativa com melhor nível de evidência, a fim de tornar os dados publicados mais confiáveis e passíveis de generalização para a aplicação no campo prático.

Quanto ao ensino em Enfermagem, é notório que o não aprendizado das habilidades básicas dessa disciplina, associado à inexperiência, pode repercutir no aumento do risco de eventos adversos aos pacientes. Logo, é evidente que os estudantes de Enfermagem podem contribuir para o acontecimento de episódios indesejados, mas evitáveis, nos serviços de saúde, como morte, lesão, incapacidade ou atraso no tratamento dos pacientes, devido a erros executados durante a assistência à saúde (41, 42).

Tal possibilidade tem potencial para gerar medo, ansiedade, sentimento de culpa e tristeza entre os estudantes de Enfermagem, afetando negativamente o seu bem-estar. Também pode diminuir a motivação e o sucesso durante o transcorrer do curso, levar à alienação ou ao afastamento da profissão ou, até mesmo, resultar na saída da escola ou da profissão ainda durante o primeiro ano (42). No entanto, a busca por melhores práticas de ensino pode tornar esse processo mais prazeroso e facilitar a aprendizagem.

Destarte, denota-se que, ao oferecer a todos os estudantes uma oportunidade ativa de aprendizado, é possível proporcionar a experimentação de experiências consistentes e comparáveis, além de ajudar a integrar os conhecimentos teórico-práticos e as habilidades. Ademais, é imprescindível que os estudantes tenham a oportunidade de repetir o treinamento com frequência, a fim de reduzir o número de erros na prática clínica e garantir a segurança do atendimento ao paciente (43).
Nesse panorama, lança-se a proposta de proporcionar uma educação que envolva a segurança no cuidado em saúde aos estudantes da graduação em Enfermagem, de modo a favorecer 0 desenvolvimento de saberes e fazeres específicos, independentemente da formação técnica pretendida. Esse processo educativo precisa estar presente em suas abordagens clínicas e na demonstração das melhores práticas, devendo ser permanente durante o desenvolvimento dos estudantes nos diferentes cenários que contribuem para a sua formação $(9,10,44)$.

Diante da necessidade de atender às demandas de aprendizagem dos estudantes de Enfermagem com o fim de contribuir para o cuidado seguro na assistência à saúde, tem-se a consolidação de metodologias inovadoras voltadas ao ensino, as quais, como pontua autor da área (45), possibilitam novas estratégias, mais dinâmicas e participativas, que posicionam os estudantes como atores ativos na construção de seu próprio conhecimento, apoiados por ferramentas educacionais inovadoras, como simulações, filmes, vídeos, encenações, dramatizações, entre outras.

O cenário da prática assistencial simulada em laboratório ou da simulação, o qual sobressaiu entre as metodologias inovadoras utilizadas nos estudos analisados na presente pesquisa, pode ser definido como uma tentativa de simular determinada situação clínica real. A simulação é um processo de educação cognitiva e comportamental que recria uma situação real em um ambiente artificial, com 0 intuito de promover uma aprendizagem significativa ao estudante, livre de danos ao paciente, com custo reduzido e maior segurança e eficácia no desenvolvimento de tarefas antes nunca realizadas pelo estudante (45-48).

0 principal objetivo da utilização da simulação no ambiente de ensino voltado à segurança do paciente é criar ambientes reais para que os estudantes possam atuar e construir seu aprendizado simulando a execução de determinado cuidado ou ação, quantas vezes forem necessárias, seja em manequim, seja em paciente simulado, a fim de aperfeiçoar suas habilidades técnicas e gerenciais $(46,48,49)$.

A formação em simulação como método de ensino inovador desempenha um papel de suma importância na aprendizagem dos estudantes de Enfermagem. Assim, o processo formativo embasado em competências clínicas pode efetivar-se mediante a possibilidade de treinar o estudante lançando mão de técnicas e procedimentos relacionados à disciplina da Enfermagem, de 
modo seguro, e com o apoio de docentes capacitados e disponíveis para fornecer um retorno imediato. Ademais, ao experimentar a vivência nos diversos cenários da prática, os estudantes podem visualizar, executar e atribuir uma (re)significação ao que se dispuseram a aprender (50).

A simulação oferece uma oportunidade para amenizar as limitações e variações nas experiências de aprendizado dos estudantes, que ocorrem durante os estágios clínicos, porquanto fornece acesso a "pacientes" padronizados de complexidade variável, de maneira a oferecer oportunidades aos estudantes de aplicar e integrar o pensamento crítico, o conhecimento e as habilidades, dentro de uma variedade de contextos e situações clínicas simuladas (51).

Posto isso, constata-se que o treinamento fundamentado em cenário de prática assistencial simulada em laboratório é capaz de melhorar os conhecimentos e as habilidades profissionais dos estudantes, aumentar a sensibilidade e a atenção para um comportamento direcionado a evitar erros nos serviços de assistência à saúde e a melhorar a qualidade e a segurança do paciente na assistência fornecida, o que, por consequência, colabora substancialmente para reduzir a taxa de eventos adversos evitáveis em suas práticas (22).

Embora essa aproximação dos estudantes com a realidade da assistência de Enfermagem por meio da simulação clínica seja fundamental, ainda existem certos desafios, a começar pelo treinamento dos docentes/educadores e dos centros de ensino na busca pela excelência do ensino de Enfermagem, cujo intuito final é formar enfermeiros que atendam às DCN brasileiras e que possam exercer suas habilidades de forma plena e com qualidade no cuidado e na assistência de Enfermagem (48).

No tocante ao uso de vídeos e filmes como apoio ao ensino da segurança do paciente na graduação em Enfermagem, trata-se de tecnologias multimídias, as quais permitem demonstrar aos estudantes um conjunto de técnicas audiovisuais que facilitam uma maior e mais rápida compreensão e interpretação das ideias. Além do mais, proporcionam um ambiente de aprendizado mais sensorial de forma a garantir que as informações aprendidas possam ser retidas por períodos mais longos $(52,53)$.

Apesar de os recursos multimídias apresentarem inúmeras possibilidades e potencialidades atreladas a estratégias de ensino sobre a segurança do paciente, o seu uso dispõe de algumas li- mitações, como a falta de feedback dos questionamentos e das dúvidas dos estudantes e a possibilidade de problemas técnicos no decurso do uso (54).

Quanto à utilização da encenação/dramatização no ensino da segurança do paciente aos estudantes do ensino superior em Enfermagem, entende-se que é uma maneira válida e eficaz de reproduzir, com segurança, situações clínicas complexas. Essa metodologia de ensino pode colaborar para uma melhor preparação dos estudantes e torná-los mais confiantes para atender pacientes em configurações reais $(24,26,55)$.

As metodologias inovadoras podem auxiliar expressivamente o ensino teórico-prático da segurança do paciente aos estudantes em Enfermagem, conferindo-Ihes a oportunidade de desenvolverem com maior efetividade e eficácia os conhecimentos e as habilidades na temática, o que reduziria os índices de eventos adversos durante os cuidados prestados ao paciente. Isso porque uma das motivações para o advento de incidentes durante a assistência à saúde é a insuficiência ou a fragilidade dos componentes conhecimentos e habilidades do fator humano (56).

Além do desenvolvimento de competências na formação dos futuros profissionais de Enfermagem, para que o cuidado seja efetivamente seguro, também se devem considerar o processo e a estrutura disponível nos serviços de saúde, tendo em vista que a ocorrência de eventos adversos é um fenômeno de ordem multifatorial e multicausal, bem como está intrinsecamente relacionada a falhas no sistema de saúde.

Nesse contexto, compreende-se que 0 ensinar e 0 aprender por meio de metodologias inovadoras não dependem somente de como a tecnologia é usada, ou seja, não adianta apenas trocar as tecnologias já utilizadas por elementos digitais de última geração, se não houver ponderação sobre as estratégias e os conteúdos dinamizados para sua utilização. Daí, denota-se o planejamento docente como elemento fundamental para tornar o processo de ensino e aprendizagem mais fluído e efetivo (56).

Logo, é significativamente importante levar em consideração que a utilização da tecnologia de maneira isolada não garante uma melhor aprendizagem, já que há a necessidade de se desenvolverem ações pedagógicas que possibilitem um fazer crítico vinculado à realidade, construído na autonomia e na cooperação dos estudantes (15). 
Esta scoping review foi realizada com enfoque específico no ensino da graduação em Enfermagem. Portanto, recomenda-se que estudos que incluam outras áreas da saúde sejam realizados, de maneira a tomar como escopo uma abordagem multiprofissional, que considere os profissionais já atuantes na prática assistencial e/ou gerencial.

\section{Conclusão}

Conclui-se que as metodologias inovadoras utilizadas para 0 ensino efetivo da segurança do paciente na graduação em En- fermagem foram simulação, vídeos, encenação/dramatização e filmes, todas aplicadas na modalidade de ensino presencial.

Além do mais, observou-se que o uso de metodologias inovadoras voltadas ao ensino do cuidado seguro na graduação em Enfermagem, com destaque para o cenário de prática assistencial simulada em laboratório, o qual foi predominante nesta scoping review e apresentou grande potencial para a efetividade e eficácia do processo de ensino-aprendizagem do tema.

Conflitos de interesses: nenhum declarado.

\section{Referências}

1. Gonçalves N, Siqueira LDC, Caliri MHL. Teaching patient safety in undergraduate courses: a bibliometric study. Rev enferm UERJ. 2017;25:e15460. DOI: http://dx.doi.org/10.12957/reuerj.2017.15460

2. Mendes W, Pavão ALB, Martins M, Moura MLO, Travassos C. The feature of preventable adverse events in hospitals in the State of Rio de Janeiro, Brazil. Rev Assoc Med Bras. 2013;59(5):421-8. DOI: https://doi.org/10.1016/j.ramb.2013.03.002

3. Sousa P, Uva AS, Serranheira F, Nunes C, Leite ES. Estimating the incidence of adverse events in Portuguese hospitals: a contribution to improving quality and patient safety. BMC Health Serv Res. 2014;14:311. DOI: https://doi. org/10.1186/1472-6963-14-311

4. Jha AK, Plaizier NP, Larizgotia I. Patient safety research: an overview of the global evidence. Qual Saf Health Care. 2010;19(1):42-7. DOI: https://doi.org/10.1136/qshc.2008.029165

5. Siman AG, Braga LM, Amaro MOF, Brito MJM. Desafios da prática na segurança do paciente. Rev Bras Enferm. 2019;72(6):1581-8. DOI: https://doi.org/10.1590/0034-7167-2018-0441

6. Rosse FV, Bruijne M, Suurmond J, Essink-Bot ML, Wagner C. Language barriers and patient safety risks in hospital care: a mixed methods study. Int J Nurs Stud. 2016;54:45-53. DOI: https://doi.org/10.1016/j.ijnurstu.2015.03.012

7. Tella S, Liukka M, Jamookeeah D, Smith NJ, Partanen P, Turunen H. What do nursing students learn about patient safety? An integrative literature review. J Nurs Educ. 2014;53(1):7-13. DOI: https://doi.org/10.3928/01484834-20131209-04

8. Lobos BM; Vergara NF. Efecto de un programa de intervención educativa sobre el conocimiento de seguridad de pacientes en estudiantes de pregrado de enfermería. Ciencia y Enfermería. 2017;23(1):97-108. DOI: https://doi.org/10.4067/ S0717-95532017000100097

9. Ministério da Saúde do Brasil. Portaria n. ${ }^{\circ}$ 529, de 1 de abril de 2013. Institui o Programa Nacional de Segurança do Paciente (PNSP). Diário Oficial da União; [citado 2 abril 2019]. Disponível em: http://bvsms.saude.gov.br/bvs/saudelegis/ gm/2013/prt0529_01_04_2013.html

10. World Health Organization (WHO). WHO patient safety curriculum guide: multi-professional edition. WHO Library Cataloguing-in-Publication. Ginebra: WHO; 2011 [citado 2 abril 2019]. Disponível em: https://apps.who.int/iris/bitstream/ handle/10665/44641/9789241501958_eng.pd;jsessionid=48BB6AA78E864680938E7B695992E339?sequence=1

11. Lee NJ, Jang H, Park SY. Patient safety education and baccalaureate nursing students' patient safety competency: A cross-sectional study. Nurs Health Sci. 2016;18(2):163-71. DOI: https://doi.org/10.1111/nhs.12237

12. Winters JRF, Prado ML, Waterkemper R, Kempfer SS. Dialogical and participative training in nursing education: contribution to the development of critical and reflective and creative thinking of students. Rev Min Enferm. 2017;21:e-1067. DOI: https://doi.org/10.5935/1415-2762.20170077 
13. Conselho Nacional de Educação do Brasil. Resolução n. ${ }^{0} 3$, de 7 de novembro de 2001 . Institui diretrizes curriculares nacionais do curso de graduação em enfermagem. Diário Oficial da União; 2001 [citado 2 abril 2019]. Disponível em: http://portal.mec.gov.br/cne/arquivos/pdf/CES03.pdf

14. Domingues AN, Tibes CM, Dias JD, Westin UM, Zem-Mascarenhas SH, Fonseca LMM. Virtual simulation by computer on nursing teaching: experience report. Rev Enferm UFPI. 2017;6(4):70-4. DOI: https://doi.org/10.26694/2238-7234.6470-74

15. Silveira MS, Cogo ALP. Contribuições das tecnologias educacionais digitais no ensino de habilidades de enfermagem: revisão integrativa. Rev Gaúcha Enferm. 2017;38(2):e66204. DOI: https://doi.org/10.1590/1983-1447.2017.02.66204

16. Villardi ML, Cyrino EG. Inovações pedagógicas no ensino superior: a problematização e o portfólio na formação de pedagogos. Revista de Estudos Aplicados em Educação. 2018;5(6):16-28. DOI: https://doi.org/10.13037/rea-e.vol3n6.5412

17. Cogo ALP, Pedro ENR, Silva APSS, Alves EATD, Valli GP. The use of digital educational technologies in nursing education. Cienc Enferm. 2013 [citado 2 abril 2019];19(3):21-9. Disponível em: http://www.scielo.br/pdf/rgenf/v38n2/en_01026933-rgenf-1983-144720170266204.pdf

18. Holanda VR, Pinheiro AKB. Comparison of learning strategies in face-to-face and online courses on sexually transmitted diseases. Texto Contexto Enferm. 2015;24(2):530-8. DOI: https://doi.org/10.1590/0104-07072015002402014

19. Joanna Briggs Institute. Joanna Briggs Institute Reviewers' Manual: 2015 edition / Supplement. South Austrália: The University of Adelaide; 2015 [citado 25 jan. 2019]. Disponível em: https://nursing.lsuhsc.edu/JBI/docs/ReviewersManuals/Scoping-.pdf

20. Arksey H, O’Malley L. Scoping studies: towards a methodological framework. Int J Soc Res Methodol. 2005;8(1):19-32. DOI: https://doi.org/10.1080/1364557032000119616

21. Joanna Briggs Institute. Levels of Evidence. South Austrália: The University of Adelaide; 2013 [citado 25 jan. 2019 ]. Disponível em: https://joannabriggs.org/sites/default/files/2019-05/JBI-Levels-of-evidence_2014_0.pdf

22. Kahriman I, Ozturk H, Bahcecik N, Sokmen S, Kucuk S, Calbayram N et al. The effect of theoretical and simulation training on medical errors of nurse students in Karadeniz Technical University, Turkey. J Pak Med Assoc. 2018 [citado 25 jan. 2019];68(11):1636-43. Disponível em: https://jpma.org.pk/article-details/8928?article_id = 8928

23. Jarvill M, Kelly S, Krebs H. Effect of expert role modeling on skill performance in simulation. Clin Simulat Nursing. 2018;24:25-9. DOI: https://doi.org/10.1016/j.ecns.2018.08.005

24. Furnes M, Kvaal KS, Hoye S. Communication in mental health nursing - Bachelor Students' appraisal of a blended learning training programme - An exploratory study. BMC Nursing. 2018;17:20. DOI: https://doi.org/10.1186/s12912018-0288-9

25. Bahar A, Arslan M, Gokgoz N, Ak H, Kaya H. Do Parenteral Medication Administration Skills of Nursing Students Increase with Educational Videos Materials? Internat J Caring Sci. 2017 [citado 18 jun. 2019];10(3):1514-25. Disponível em: http://www.internationaljournalofcaringsciences.org/docs/45_bahar_original_10_3.pdf

26. Hayes C, Jackson D, Davidson PM, Daly J, Power T. Calm to chaos: Engaging undergraduate nursing students with the complex nature of interruptions during medication administration. J Clin Nurs. 2017;26(23-24):4839-47. DOI: https:// doi.org/10.1111/jocn.13866

27. Abelsson A, Bisholt B. Nurse students learning acute care by simulation - Focus on observation and debriefing. Nurse Educ Pract. 2017;24:6-13. DOI: https://doi.org/10.1016/j.nepr.2017.03.001

28. Lobos BM, Vergara NF. Effect of an educational intervention program on patient safety knowledge among undergraduate nursing students. Cienc. Enferm. 2017;23(1):97-108. DOI: https://doi.org/10.1186/s12909-018-1255-6

29. Breitkreuz KR, Dougal RL, Wright MC. How do simulated error experiences impact attitudes related to error prevention? Simul Healthc. 2016;11(5):323-33. DOI: https://doi.org/10.1097/SIH.0000000000000174

30. Forbes H, Bucknall TK, Hutchinson AM. Piloting the feasibility of head-mounted video technology to augment student feedback during simulated clinical decision-making: An observational design pilot study. Nurse Educ Today. 2016;39:116-21. DOI: https://doi.org/10.1016/j.nedt.2016.01.012 
31. Grant JS, Dawkins D, Molhook L, Keltner NL, Vance DE. Comparing the effectiveness of video-assisted oral debriefing and oral debriefing alone on behaviors by undergraduate nursing students during high-fidelity simulation. Nurse Educ Pract. 2014;14(5):479-84. DOI: https://doi.org/10.1016/j.nepr.2014.05.003

32. Holland A, Smith F, McCrossan G, Adamson E, Watt S, Penny K. Online video in clinical skills education of oral medication administration for undergraduate student nurses: A mixed methods, prospective cohort study. Nurse Educ Today. 2013;33(6):663-70. DOI: https://doi.org/10.1016/j.nedt.2012.01.006

33. Nevin M, Neill F, Mulkerrins J. Preparing the nursing student for internship in a pre-registration nursing program: Developing a problem based approach with the use of high fidelity simulation equipment. Nurse Educ Pract. 2014;14(2):1549. DOI: https://doi.org/10.1016/j.nepr.2013.07.008

34. Grugnetti AM, Bagnasco A, Rosa F, Sasso L. Effectiveness of a clinical skills workshop for drug-dosage calculation in a nursing program. Nurse Educ Today. 2014;34(4):619-24. DOI: https://doi.org/10.1016/j.nedt.2013.05.021

35. Pinar G, Dogan N. Improving perinatal patient safety among turkish nursing students using simulation training. Procedia. 2013;83:88-93. DOI: https://doi.org/10.1016/j.sbspro.2013.06.017

36. Davies J, Nathan M, Clarke D. An evaluation of a complex simulated scenario with final year undergraduate children's nursing students. Collegian. 2012;19(3):131-8. DOI: https://doi.org/10.1016/j.colegn.2012.04.005

37. Husebø SE, Rystedt H, Friberg F. Educating for teamwork - nursing students' coordination in simulated cardiac arrest situations. J Adv Nurs. 2011;67(10):2239-55. DOI: https://doi.org/10.1111/j.1365-2648.2011.05629.x

38. Endacott R, Scholes J, Buykx P, Cooper S, Kinsman L, McConnell-Henry T. Final-year nursing students' ability to assess, detect and act on clinical cues of deterioration in a simulated environment. J Adv Nurs. 2010;66(12):2722-31. DOI: https://doi.org/10.1111/j.1365-2648.2010.05417.x

39. Johannesson E, Olsson MO, Petersson G, Silén C. Learning features in computer simulation skills training. Nurse Educ Pract. 2010;10(5):268-73. DOI: https://doi.org/10.1016/j.nepr.2009.11.018

40. Gantt LT, Webb-Corbett R. Using simulation to teach patient safety behaviors in undergraduate nursing education. J Nurs Educ. 2010;49(1):48-51. DOI: https://doi.org/10.3928/01484834-20090918-10

41. Yilmaz DU, Korhan EA. Effectiveness of simulation methods in nursing education: A systematic review. BMC Med Educ. 2017;9:218-26. DOI: https://doi.org/10.1186/s12909-016-0672-7

42. Cebeci F, Karazeybek E, Sucu G, Kahveci R. Nursing students' medication errors and their opinions on the reasons of errors: A cross-sectional survey. J Pak Med Assoc. 2015 [citado 18 jun. 2019];65:457-62. Avaialable from: https://www. researchgate.net/publication/277560127

43. Edeer DA, Sarikaya A. The Use of Simulation in nursing education and simulation types. J Educ Res Nurs. 2015;12:1215. DOI: https://doi.org/10.1186/s12909-016-0672-7

44. Bohomol E, Freitas MAO, Cunha ICKO. Ensino da segurança do paciente na graduação em saúde: reflexões sobre saberes e fazeres. Interface Comun Saúde Educ. 2016;20(58):727-41. DOI: https://doi.org/10.1590/1807-57622015.0699

45. Mazzo A, Martins JCA, Baptista RCN, Godoy S, Coutinho VRD, Seixas CA et al. A simulação e a videoconferência no ensino de enfermagem. Rev Grad USP. 2017;2(2):55-63. DOI: https://doi.org/10.11606/issn.2525-376X.v2i2p55-63

46. Carvalho ECC. A look at the non-technical skills of nurses: Simulation contributions. Rev Latino-Am Enfermagem. 2016;24:e2791. DOI: https://doi.org/10.1590/1518-8345.0000.2791

47. Martins JCS. Learning and development in simulated practice environments. Rev Enf Ref. 2017;IV(12):155-61. DOI: https://doi.org/10.12707/RIV16074

48. Silveira RCP, Silva FM. O uso da web e a simulação buscando a excelência no ensino de enfermagem. Rev Enf UFJF. 2016;2(1):57-62. Disponível em: https://periodicos.ufjf.br/index.php/enfermagem/article/view/3842

49. Engströom H, Hagiwara AM, Backlund P, Lebram M, Lundberg L, Johannesson M et al. The impact of contextualization on immersion in healthcare simulation. Adv. Simul. 2016;1:8. DOI: https://doi.org/10.1186/s41077-016-0009-y 
50. Costa RRO, Medeiros SM, Vitor AF, Lira ALBC, Martins JCA, Araujo MS. Tipos e finalidades da simulação no ensino de graduação em enfermagem: revisão integrativa da literatura. Rev. baiana enferm. 2016;30(3):1-11. DOI: https://doi. org/10.18471/rbe.v30i3.16589

51. Motola I, Devine LA, Chung HS, Sullivan JE, Issenberg BS. Simulation in healthcare education: A best evidence practical guide. Med Teach. 2013;35(10):e1511-30. DOI: https://doi.org/10.3109/0142159X.2013.818632

52. Sowan AK. Multimedia applications in nursing curriculum: the process of producing streaming videos for medication administration skills. Int J Med Inform. 2014;83(7):529-35. DOI: https://doi.org/10.1016/j.ijmedinf.2014.04.004

53. Cooper E. Nursing student medication errors: a snapshot view from a school of nursing's quality and safety officer. J Nurs Educ. 2014;53(3):S51-4. DOI: https://doi.org/10.3928/01484834-20140211-03

54. Delen E, Liew J, Willson V. Effects of interactivity and educational scaffolding on learning: Self-regulation in online videobased environments. Computers \& Education. 2014;78:312-20. DOI: https://doi.org/10.1016/j.compedu.2014.06.018

55. Lubbers J, Rossman C. The effects of pediatric Community simulation experience on the self-confidence and satisfaction of baccalaureate nursing students: A quasi-experimental study. Nurse Educ Today. 2016;39:93-8. DOI: https://doi. org/10.1016/j.nedt.2016.01.013

56. Saygin T, Keklik B. A study about causes of medical errors: Isparta Province Sample. Hacettepe Journal of Health Administration. 2014;17(2):99-118. 